Оригинальная статья/Original article

УДК 664.788.4:66-9

DOI: http://doi.org/10.20914/2310-1202-2016-2-11-15

Исследование теплофизических характеристик зерна проса,

высушенного в аппарате с закрученными потоками

теплоносителя и СВЧ-энергоподводом

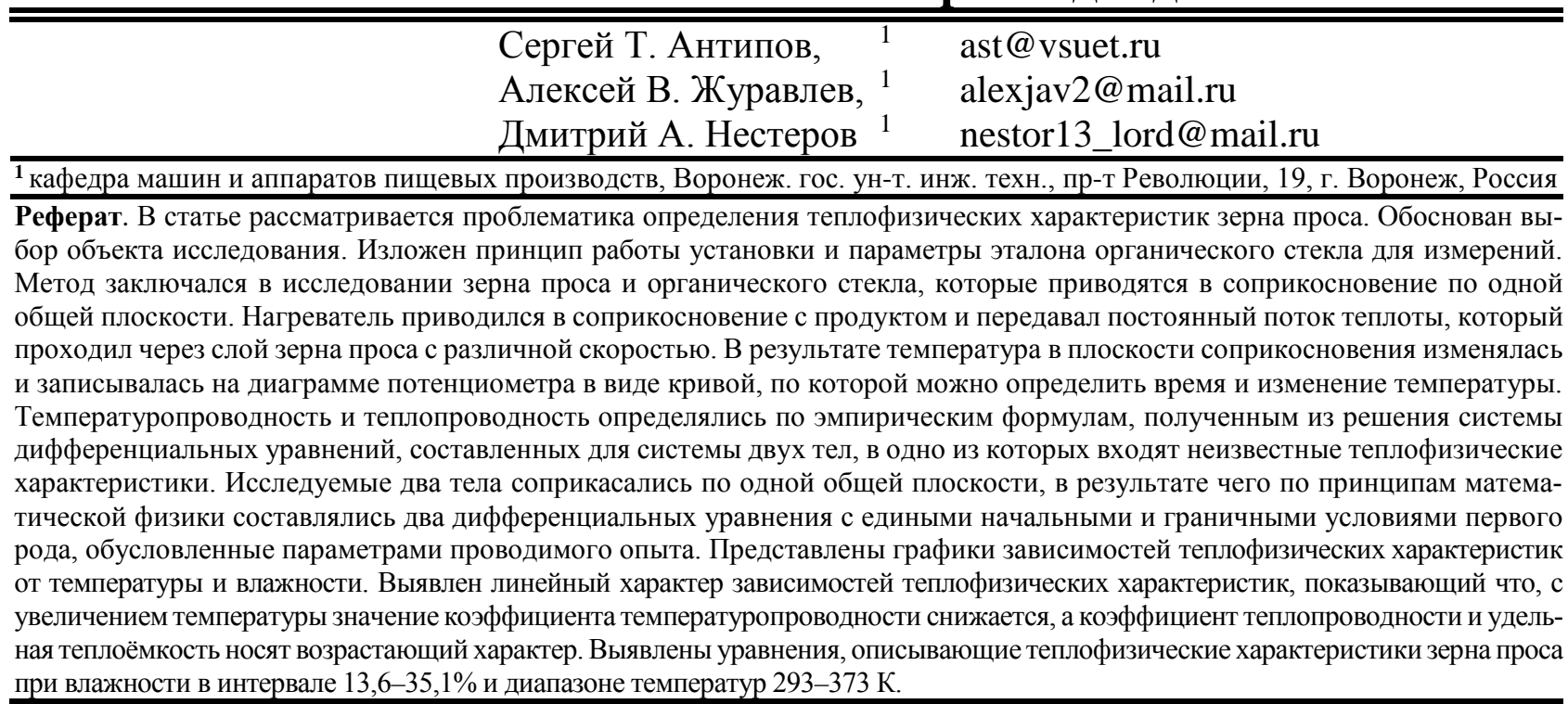

Ключевые слова: сушка, зерно проса, теплофизические характеристики, удельная теплоёмкость, теплопроводность, коэффициент температуропроводности.

\title{
Study thermal characteristics of millet grain, dried in the machine with the swirling flow of the coolant and the microwave energy supply
}

$\begin{array}{lll}\text { Sergei T. Antipov, } & 1 & \text { ast@vsuet.ru } \\ \text { Aleksey V. Zhuravlev, } & 1 & \text { alexjav2@mail.ru } \\ \text { Dmitrii A. Nesterov } & 1 & \text { nestor13_lord@mail.ru }\end{array}$

$\overline{1}$ machinery and equipment for food production department , Voronezh state university of engineering technologies, Revolution Av., 19, Voronezh, Russia

Summary. The article discusses the problems of determining the thermal characteristics of millet. The choice of the research object. The paper presents the principle of operation of the plant and the parameters of the standard, organic glass for measurements. Method was to study millet grains and organic glass, which are brought into contact on a common plane. The heater is brought into contact with the product and passed the constant heat flow, which passed through a layer of millet grain at different speeds. As a result, the temperature in the contact plane of the changed and recorded on the chart of the potentiometer in the form of the curve, by which you can determine the time and temperature change. The thermal diffusivity and thermal conductivity determined by empirical formulas obtained by solving a system of differential equations, made up for the system of two bodies, one of which includes the unknown thermal characteristics. Test two bodies in contact on a common plane, resulting in mathematical physics principles constitute two differential equations with uniform initial and boundary conditions of the first kind, due to the parameters of ongoing experience. It is a plot of thermal performance of the temperature and humidity. Revealed linear dependence of the physical thermal characteristics, showing that with increasing temperature the thermal diffusivity value decreases, and the thermal conductivity and specific heat capacity are increasing character. Revealed the equations describing the thermal characteristics of millet grain with a humidity in the range of $13.6-35.1 \%$ and the temperature range $293-373 \mathrm{~K}$.

Keywords: drying, grain millet, thermal characteristics, specific heat, thermal conductivity, thermal diffusivity.

Для цитирования

Антипов С. Т., Журавлев А. В., Нестеров Д. А.Исследование теплофизических характеристик зерна проса, высушенного в аппарате с закрученными потоками теплоносителя и СВЧ-энергоподводом // Вестник ВГУИТ. 2016. № 2. С 11-15. doi:10.20914/2310-1202-2016-2-11-15
For citation

Antipov S. T, Zhuravlev A. V., Nesterov D. A. Study thermal characteristics of millet grain, dried in the machine with the swirling flow of the coolant and the microwave energy supply. Vestnik VSUET [Proceedings of VSUET]. 2016. no. 2 pp. 11-15. (in Russ.). doi:10.20914/2310-12022016-2-11-15 


\section{Введение}

Исследование теплофизических характеристик продукта является важной составляющей для научного обоснования процесса сушки, а также создания нового сушильного оборудования или адаптации к уже существующим аппаратам [1]. В качестве объекта исследования нами было выбрано зерно проса, как обладающее повышенными физико-химическими показателями [2]. Сушка производилась в разработанном на кафедре МАПП Воронежского государственного университета инженерных технологий аппарате с закрученными потоками теплоносителя и СВЧ-энергоподводом [3, 4].

\section{1 Материалы и методы}

При определении теплофизических характеристик, таких как удельная теплоёмкость $c$, Дж/(кг К К), коэффициент температуропроводности $a, \mathrm{~m}^{2} / \mathrm{c}$, коэффициент теплопроводности $\lambda, \mathrm{BT} /(\mathrm{M} \cdot \mathrm{K})$, использован метод разработанный В. С. Волькенштейн, основанный на решении задачи теплопроводности для начальной стадии процесса, а именно метод двух температурно-временных точек.

Определение теплофизических характеристик зерна проса производилось на установке Cossfield RT-1394H.

Метод, применяемый в данной установке, заключается в исследовании ограниченного (пищевой продукт) и полуограниченного (эталон) тел, которые приводятся в соприкосновение по одной общей плоскости. Нагреватель, нагретый до постоянной температуры, приведён в соприкосновение с продуктом и передаёт постоянный поток теплоты, который проходит через толщину пищевого продукта с различной скоростью. В результате температура в плоскости соприкосновения пищевого продукта с эталоном изменяется и записывается на диаграмме потенциометра в виде кривой, по которой можно определить время и изменение температуры.

Начальная температура продукта и эталона в течение всего опыта поддерживалась постоянной. Эталон с сечением $70 \times 70$ мм и длиной 150 мм изготовлен из органического стекла и имеет следующие характеристики: $\alpha_{3}=15,7 \mathrm{~m}^{2} / \mathrm{c}, \lambda_{3}=$ $0,1839 \mathrm{BT} / \mathrm{M} \cdot \mathrm{K}, c_{\ni}=10,88$ Дж/Кг $\cdot \mathrm{K}, \rho_{3}=1075 \mathrm{\kappa} \Gamma / \mathrm{M}^{3}$.

Во время эксперимента фиксируется время $\tau_{1}$ и $\tau_{2}$, в течение которого температура на границе раздела эталонного тела и исследуемого образца достигнет заданного значения $\Delta T_{1}$ и $\Delta T_{2}$ соответственно, разница при этом в нашем эксперименте составляла $20 \mathrm{~K}$.

Управление ходом эксперимента и обработка измерительной информации осуществляется посредством виртуального прибора, разработанного в LabView 7.0.
Температуропроводность и теплопроводность определяются по эмпирическим формулам, полученным из решения системы дифференциальных уравнений, составленных для системы двух тел, в одно из которых входят неизвестные теплофизические характеристики [5]. Относительно этих характеристик решается система уравнений, включающая параметры проведённого опыта. Так как исследуемые два тела соприкасаются по одной общей плоскости, то по принципам математической физики можно составить два дифференциальных уравнения с едиными начальными и граничными условиями первого рода, обусловленных параметрами проводимого опыта [6, 7].

Температуропроводность, Вт/(м.К) исследуемого образца пищевого продукта определяется по эмпирической формуле

$$
\lambda_{M}=\lambda_{\ni} \frac{1-h}{1+h} \sqrt{\frac{a_{M}}{a_{\vartheta}}},
$$

где $\lambda_{\mu}, a_{\mu} ; \lambda_{3}, a_{3}$ - соответственно теплопроводность и температуропроводность образца исследуемого пищевого продукта и эталона; $h-$ вспомогательная величина, определяемая по формуле:

$$
h=\frac{t^{\prime \prime}}{t_{z p}\left[1-\Phi\left(z^{\prime \prime}\right)\right]}-1,
$$

где $t^{\prime \prime}$ - температура в плоскости соприкосновения образца продукта и эталона, определяемая по полученной диаграмме, ${ }^{\circ} \mathrm{C} ; t_{2 p}$-температура греющей поверхности, определяемая по полученной диаграмме аналогично, ${ }^{\circ} \mathrm{C} ; \Phi\left(z^{\prime \prime}\right)$ - функция Гаусса.

Удельная теплоёмкость пищевого продукта, Дж/(кг·К) определяется по зависимости:

$$
c_{M}=\lambda_{M} /\left(a_{\mu} \rho_{\mu}\right),
$$

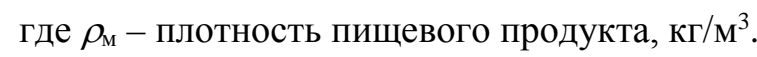

\section{2 Результаты и обсуждение}

Зависимости теплофизических характеристик зерна проса представлены на рисунках 1-6.

Данные эксперимента были обработаны при помощи программной среды «Table Curve 3D», позволяющей получить зависимости 4-6 теплофизических характеристик от влагосодержания в интервале $13,6-35,1 \%$ и диапазоне температур 293-373 K.

$$
\begin{aligned}
& \alpha=250,12-76,69 \cdot \operatorname{Ln} T+0,58 \cdot W_{0}^{c}+6 \cdot(\operatorname{Ln} T)^{2} ; \\
& \lambda=\left(0,17+1,23 \cdot T+1,95 \cdot W_{0}^{c 2}\right) /\left(1+7,18 \cdot T^{2}\right) ; \\
& c=28433,55-260,19 \cdot T-142,96 \cdot W_{0}^{c}+ \\
& +0,8 \cdot T^{2}-1,06 \cdot W_{0}^{c 2}+1,05 \cdot T \cdot W_{0}^{c}
\end{aligned}
$$




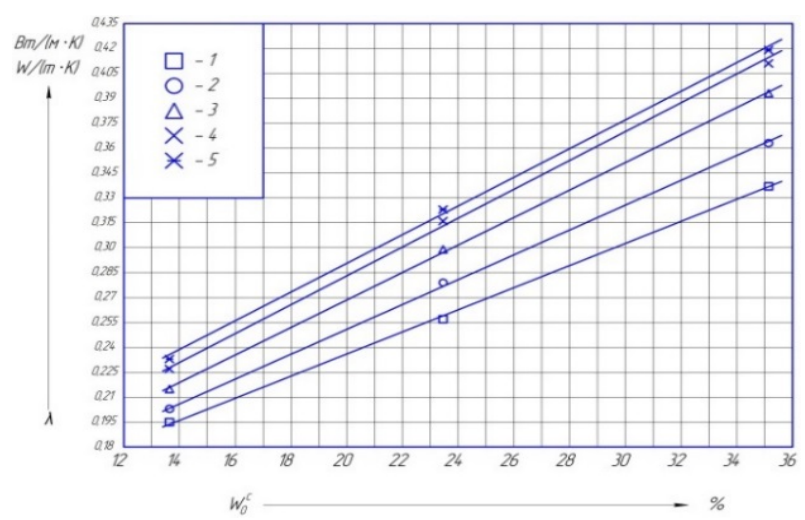

Рисунок 1. Зависимость коэффициента теплопроводности $\lambda, \mathrm{BT} /(\mathrm{м} \cdot \mathrm{K})$, от влажности при: 1 - Т = $293 \mathrm{~K} ; 2$ - Т = $313 \mathrm{~K}$; $3-\mathrm{T}=333 \mathrm{~K} ; 4-\mathrm{T}=353 \mathrm{~K} ; 5-\mathrm{T}=373 \mathrm{~K}$

Figure 1 . The dependence of the thermal conductivity $\lambda$, $\mathrm{W} /(\mathrm{m} \cdot \mathrm{K})$, from the humidity: $1-\mathrm{T}=293 \mathrm{~K} ; 2-\mathrm{T}=313 \mathrm{~K}$; $3-\mathrm{T}=333 \mathrm{~K} ; 4-\mathrm{T}=353 \mathrm{~K} ; 5-\mathrm{T}=373 \mathrm{~K}$

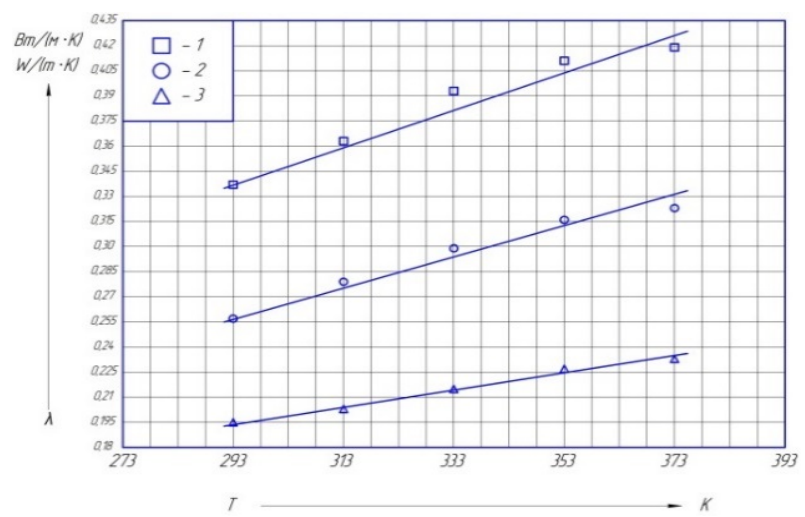

Рисунок 2. Зависимость коэффициента теплопроводности $\lambda, \mathrm{Bт} /(\mathrm{м} \cdot \mathrm{K})$, от температуры при: $1-W_{0}^{\mathrm{c}}=13,6 \%$; $2-W_{0}{ }^{\mathrm{c}}=23,5 \% ; 3-W_{0}^{\mathrm{c}}=35,1 \%$

Figure 2. The dependence of the thermal conductivity $\lambda$, $\mathrm{W} /(\mathrm{m} \cdot \mathrm{K})$, at a temperature from: $1-W_{0} \mathrm{c}=13,6 \%$; $2-W_{0}{ }^{\mathrm{c}}=23,5 \% ; 3-W_{0}{ }^{\mathrm{c}}=35,1 \%$

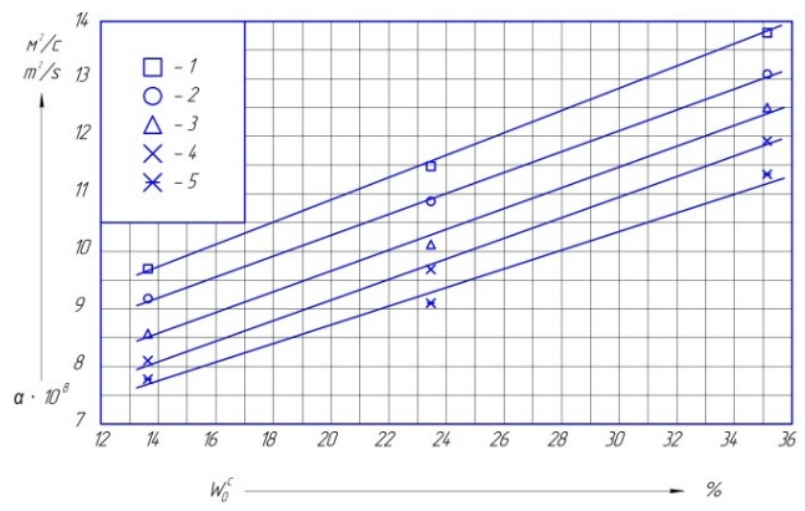

Рисунок 3. Зависимость коэффициента температуропроводности $\alpha \cdot 10^{8}, \mathrm{~m}^{2} / \mathrm{c}$, от влажности при: $1-\mathrm{T}=293 \mathrm{~K}$; $2-\mathrm{T}=313 \mathrm{~K} ; 3-\mathrm{T}=333 \mathrm{~K} ; 4-\mathrm{T}=353 \mathrm{~K} ; 5-\mathrm{T}=373 \mathrm{~K}$

Figure 3. The dependence of the thermal diffusivity coefficient $\alpha \cdot 10^{8}, \mathrm{~m}^{2} / \mathrm{s}$, from humidity: $1-\mathrm{T}=293 \mathrm{~K} ; 2-$ $\mathrm{T}=313 \mathrm{~K} ; 3-\mathrm{T}=333 \mathrm{~K} ; 4-\mathrm{T}=353 \mathrm{~K} ; 5-\mathrm{T}=373 \mathrm{~K}$

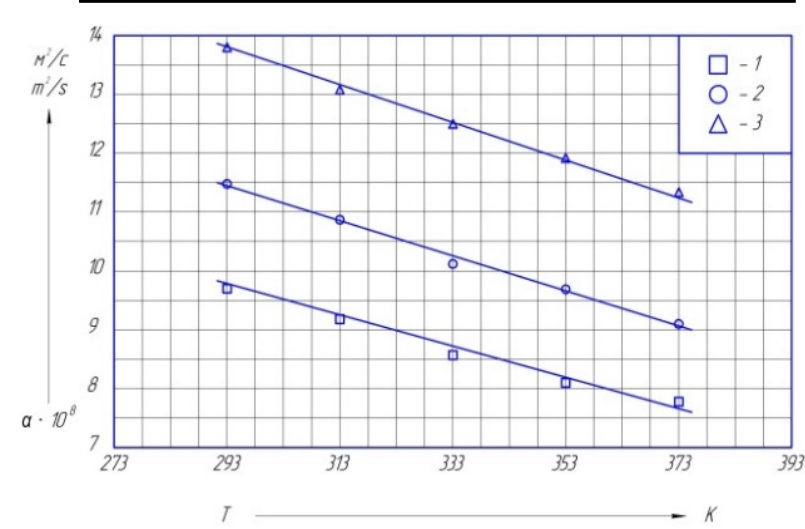

Рисунок 4. Зависимость коэффициента температуропроводности $\alpha \cdot 10^{8}, \mathrm{~m}^{2} / \mathrm{c}$, от температуры при: $1-W_{0}{ }^{\mathrm{c}}=13,6 \% ; 2-W_{0}{ }^{\mathrm{c}}=23,5 \% ; 3-W_{0} \mathrm{c}=35,1 \%$

Figure 4 . The dependence of the thermal diffusivity $\alpha \cdot 10^{8} \mathrm{~m}^{2} / \mathrm{s}$, at a temperature from: $1-W_{0}{ }^{\mathrm{c}}=13,6 \%$; $2-W_{0}{ }^{\mathrm{c}}=23,5 \% ; 3-W_{0}{ }^{\mathrm{c}}=35,1 \%$

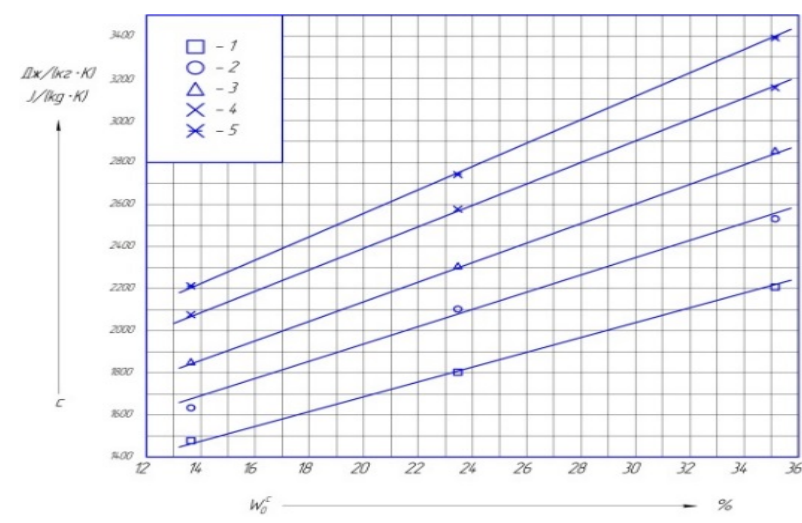

Рисунок 5. Зависимость коэффициента удельной теплоёмкости $c$, Дж/(кг $\cdot К)$ от влажности при: $1-\mathrm{T}=293 \mathrm{~K}$; $2-\mathrm{T}=313 \mathrm{~K} ; 3-\mathrm{T}=333 \mathrm{~K} ; 4-\mathrm{T}=353 \mathrm{~K} ; 5-\mathrm{T}=373 \mathrm{~K}$

Figure 5. The dependence of the specific heat coefficient $c$, $\mathrm{J} /(\mathrm{kg} \cdot \mathrm{K})$, from humidity: $1-\mathrm{T}=293 \mathrm{~K} ; 2-\mathrm{T}=313 \mathrm{~K}$; $3-\mathrm{T}=333 \mathrm{~K} ; 4-\mathrm{T}=353 \mathrm{~K} ; 5-\mathrm{T}=373 \mathrm{~K}$

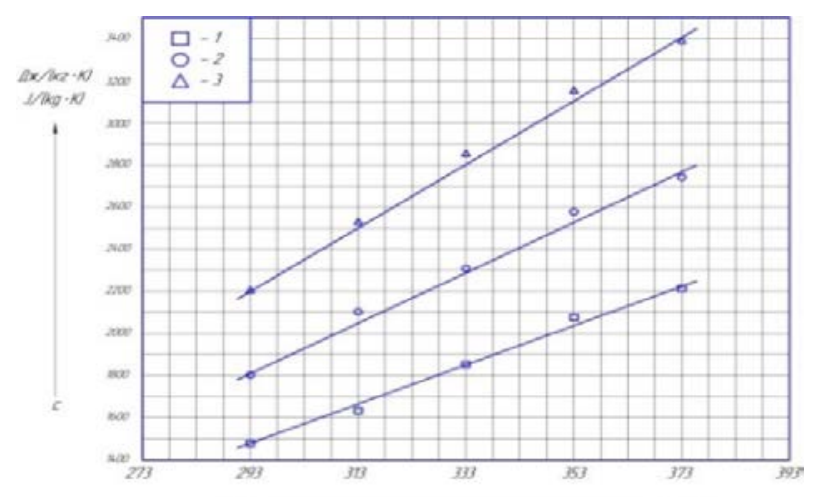

Рисунок 6. Зависимость коэффициента удельной теплоёмкости с, Дж/(кг·К) от температуры при: $1-W_{0}{ }^{\mathrm{c}}=13,6 \% ; 2-W_{0}{ }^{\mathrm{c}}=23,5 \% ; 3-W_{0}{ }^{\mathrm{c}}=35,1 \%$

Figure 6. The dependence of the specific heat coefficient $c, \mathrm{~J} /(\mathrm{kg} \cdot \mathrm{K})$, at a temperature from: $1-W_{0}{ }^{\mathrm{c}}=13,6 \%$; $2-W_{0}{ }^{\mathrm{c}}=23,5 \% ; 3-W_{0}{ }^{\mathrm{c}}=35,1 \%$ 
В ходе обработки экспериментальных данных выявлено, что теплофизические характеристики (коэффициент температуропроводности, коэффициент теплопроводности и удельная теплоёмкость) имеют линейный характер.

С увеличением температуры значение коэффициента температуропроводности снижается, а коэффициент теплопроводности

\section{ЛИТЕРАТУРА}

1 Васильев В. Н., Куцакова В. Е., Фролов С. В. Технология сушки. Основы тепло- и массообмена: учебник. СПб.: ГИОРД, 2013. 222 с.

2 Коновалов К. Л., Шулбаева М. Т. Растительные пищевые композиты для производства комбинированных продуктов // Пищевая промышленность. 2008. № 7. С. 8-10.

ЗПат. 2544406 Российская Федерация, МПК7 F 26 В 17/10, F 26 В3/347. Аппарат для сушки дисперсных материалов в закрученном потоке теплоносителя с СВЧ-энергоподводом / Казарцев Д. А., Антипов С. Т., Журавлев А. В., Нестеров Д. А., Бородкина А. В.; заявитель и патентообладатель Воронеж. гос. унив. инж. тех. № 2013150692/06; Заявл. 14.11.2013; Опубл. 20.03.2015, Бюл. № 8.9 с.

4 Антипов С. Т., Журавлев А. В., Казарцев Д. А., Бородкина А. В. и др. Комбинированные аппараты с закрученным потоком теплоносителя для сушки дисперсных материалов // Технологии пищевой и перерабатывающей промышленности АПК - продукты здорового питания. Воронеж. № 2. 2014. С. 52-59.

5 Чубик И. А., Маслов А. М. Справочник по теплофизическим характеристикам пищевых продуктов и материалов. М.: Изд-во «Книга по требованию», 2012. 185 с.

6 Sharma G. P., Prasad S. Specific energy consumption in microwave drying of garlic cloves // Energy. 2006. V. 31. №. 12. P. 1921-1926. doi:10.1016/j.energy.2005.08.006

7 Bhupender S. K., Rajneesh B., Baljeet, S. Y. Physicochemical, functional, thermal and pasting properties of starches isolated from pearl millet cultivars // International Food Research Journal. 2013. № 20(4). P. 1555-1561. и удельная теплоёмкость носят возрастающий характер, что подтверждается при подстановке полученных значений в уравнение 3 .

\section{Заключение}

Полученные данные в дальнейшем будут использованы при разработке математических моделей процесса сушки зерна проса для любых сушильных установок.

\section{REFERENCES}

1 Vasiliev V. N., Kutsakova V. E., Frolov S. V. Tekhnologiya sushki. Osnovy teplo- i massoobmena. [Drying technology. Fundamentals of Heat and Mass Transfer] Saint-Petersburg, GIORD, 2013. 222 p. (in Russian).

2 Konovalov K. L. Vegetable food composites for the production of combination products / K. L. Konovalov, Shulbaeva M. T. // Food Industry. 2008. № 7. S. 8-10. (in Russian).

3 Kazartsev D. A., Antipov S. T., Zhuravlev A. V., Nesterov D. A., Borodkina A. V. Apparat dlya sushki dispersnykh materialov $\mathrm{v}$ zakruchennom potoke teplonositelya s SVCh-energopodvodom [Apparatus for drying particulate materials in the swirling flow of coolant to the microwave energy supply] Patent RF, no. 2544406, 2015. (in Russian).

4 Antipov S. T., Zhuravlev A. V., Kazartsev D.A, Borodkina A. V. et al. Combined devices with twisted coolant flow for drying particulate materials. Tekhnologii pishchevoi i pererabatyvayushchei promyshlennosti. APK - produkty zdorovogo pitaniya [Technology of food processing industry agro-industrial complex - healthy food]. 2014, no. 2, pp. 52-59. (in Russian).

5 Chubik I. A., Maslov A. M. Spravochnik po teplofizicheskim kharakteristikam pishchevykh produktov i materialov [Handbook of thermophysical characteristics of foods and materials]. Moscow, Kniga po trebovaniyu, 2012. 185 p. (in Russian).

6 Sharma G. P., Prasad S. Specific energy consumption in microwave drying of garlic cloves. Energy. 2006, no. 12, pp. 1921-1926. doi:10.1016/j.energy.2005.08.006

7 Bhupender S. K., Rajneesh B., Baljeet S. Y. Physicochemical, functional, thermal and pasting properties of starches isolated from pearl millet cultivars . International Food Research Journal. 2013, no. 20(4), pp. 1555-1561. 


\section{СВЕДЕНИЯ ОБ АВТОРАХ}

Сергей Т. Антипов д.т.н., профессор, проректор по научной и инновационной деятельности, Воронеж. гос. ун-т. инж. техн., пр-т Революции, 19, г. Воронеж, 394036, Россия, ast@vsuet.ru

Алексей В. Журавлев к.т.н., доцент, кафедра машин и аппаратов пищевых производств, Воронеж. гос. ун-т. инж. техн., пр-т Революции, 19, г. Воронеж, 394036, Россия, alexjav2@mail.ru

Дмитрий А. Нестеров аспирант, кафедра машин и аппаратов пищевых производств, Воронеж. гос. ун-т. инж. техн., пр-т Революции, 19, г. Воронеж, 394036 , Россия, nestor13_lord@mail.ru

\section{КРИТЕРИЙ АВТОРСТВА}

Сергей Т. Антипов предложил схему проведения эксперимента, консультация в ходе испытаний

Алексей В. Журавлев консультация в ходе исследовании Дмитрий А. Нестеров написал рукопись, корректировал её до подачи в редакцию и несёт ответственность за плагиат

\section{КОНФЛИКТ ИНТЕРЕСОВ}

Авторы заявляют об отсутствии конфликта интересов.

ПОСТУПИЛА 11.03.2016

ПРИНЯТА В ПЕЧАТЬ 08.04.2016

\section{INFORMATION ABOUT AUTHORS}

Sergei T. Antipov d.t.sc, professor, vice-rector for research and innovation, Voronezh state university of engineering technologies, Revolution Av., 19 Voronezh, Russia, ast@vsuet.ru

Alexey V. Zhuravlev Ph.d., assistant professor, department of machines and equipment for food production, Voronezh state university of engineering technologies, Revolution Av., 19 Voronezh, Russia, alexjav2@mail.ru

Dmitriy A. Nesterov graduate student, department of machines and equipment for food production, Voronezh state university of engineering technologies, Revolution Av., 19 Voronezh, Russia, nestor13_lord@mail.ru

\section{CONTRIBUTION}

Sergei T. Antipov proposed a scheme of the experiment, test consulting input

Aleksey V. Zhuravlev consultation during the study

Dmitrii A. Nesterov wrote the manuscript, correct it before filing in the office and is responsible for plagiarism

\section{CONFLICT OF INTEREST}

The authors declare no conflict of interest.

RECEIVED 3.11.2016

ACCEPTED 4.8.2016 